\title{
A eficácia das ações de educação em saúde no controle e prevenção das neoplasias do
}

\section{colo do útero}

The effectiveness of health education actions in the control and prevention of cervical cancer

La eficacia de las acciones de educación sanitaria en el control y la prevención del cáncer de cuello

uterino

Recebido: 03/12/2021 | Revisado: 10/11/2021 | Aceito: 11/12/2021 | Publicado: 14/12/2021

\author{
Karine dos Santos Tiago \\ ORCID: https://orcid.org/0000-0002-7046-6784 \\ Centro Universitário Fametro, Brasil \\ E-mail: tiagokarine31@gmail.com \\ Mykaella Lee Nascimento Souza \\ ORCID: https://orcid.org/0000-0001-8842-1958 \\ Centro Universitário Fametro, Brasil \\ E-mail: kaella.souza17@gmail.com \\ Paula Figliuolo da Cruz Borges \\ ORCID: https://orcid.org/0000-0002-1323-0062 \\ Centro Universitário Fametro, Brasil \\ E-mail: paula.fcruz86@gmail.com
}

\begin{abstract}
Resumo
Objetivo: descrever a eficácia das ações de educação em saúde no controle e prevenção das neoplasias do colo do útero. Metodologia: $\mathrm{O}$ artigo utilizou a metodologia de revisão integrativa da literatura com base qualitativadescritiva, a coleta nos repositórios eletrônicos se deram a partir de agosto de 2020 à novembro de 2021, foram encontrados 1.411 artigos, após análise e exclusão daqueles que não atendiam a pesquisa utilizou-se o total de 14 . Resultados: Diferentes estudos empregam meios de comunicação heterogênicos para fomentar o conhecimento sobre o câncer cervical, bem como seus riscos, manifestações clínicas, tratamento e prevenção, como: ligações telefônicas, apresentação em slides, discussão grupais, palestras informativas, aconselhamentos, entre outros. Conclusão: As ações de educação em saúde tornam-se uma estratégia muito promissora, haja visto, que é uma atividade de baixo custo que permite a difusão de informações e conhecimento para a população em amplo aspecto, corroborando para o aumento da procura daquelas que recebem as ações de educação pela vacinação contra o HPV e a realização do exame de Papanicolau.
\end{abstract}

Palavras-chave: Educação em saúde; Prevenção de doenças; Controle; Neoplasias do colo do útero.

\begin{abstract}
Objective: To describe the effectiveness of health education actions in the control and prevention of cervical cancer. Methodology: The article used the methodology of integrative literature review with qualitative-descriptive basis, the collection in electronic repositories took place from August 2020 to November 2021, 1,411 articles were found, after analysis and exclusion of those who did not meet the research used a total of 14. Results: Different studies employ heterogeneous means of communication to foster knowledge about cervical cancer, as well as its risks, clinical manifestations, treatment and prevention, such as: phone calls, slide presentation, group discussion, informative lectures, counseling, among others. Conclusion: The actions of health education become a very promising strategy, since it is a low-cost activity that allows the dissemination of information and knowledge to the population in broad aspect, corroborating the increase in demand for those who receive the educational actions for vaccination against HPV and the Pap smear test.
\end{abstract}

Keywords: Health education; Disease prevention; Control; Uterine cervical neoplasms.

\section{Resumen}

Objetivo: Desvelar la eficacia de las acciones de educación en salud en el control y la prevención de las neoplasias del cuello del útero. Metodología: El artículo utilizó la metodología de revisión integradora de la literatura con base cualitativa-descriptiva, la cual se recogió en los repositorios electrónicos desde agosto de 2020 hasta noviembre de 2021, encontrándose 1.411 artículos, tras el análisis y exclusión de los que no respondían a la investigación se utilizó un total de 14. Resultados: Diferentes estudios emplean medios de comunicación heterogéneos para fomentar el conocimiento sobre el cáncer de cuello uterino, así como sus riesgos, manifestaciones clínicas, tratamiento y prevención, tales como: llamadas telefónicas, presentación de diapositivas, discusión grupal, charlas informativas, consejería, entre otros. Conclusión: Las acciones de educación en salud se convierten en una estrategia muy 
prometedora, ya que es una actividad de bajo costo que permite la difusión de información y conocimiento para la población en general, corroborando el aumento de la procuración de las personas que reciben las acciones de educación para la vacunación contra el VPH y la realización del examen de Papanicolau.

Palabras clave: Educación en salud; Prevención de enfermedades; Control; Neoplasias del cuello uterino.

\section{Introdução}

As neoplasias do colo do útero representam um problema de saúde pública internacional, tornando-se o terceiro tipo de câncer mais comum entre as mulheres em idade reprodutiva (Sitaresmi et al., 2020; Davilla et al., 2021). Essa patologia se dá por alterações celulares que podem desencadear o câncer, provocadas normalmente pela infecção persistente por algum subtipo do Papilomavírus Humano (HPV) (INCA, 2018). Estima-se que cerca de 569.847 novos casos do câncer de colo do útero são notificados anualmente, sendo 311.365 mortes no mundo (Sitaresmi et al., 2020). No Brasil, o câncer do colo do útero apresenta para 2020, 2021 e 2022 uma estimativa de 16.590 novos casos para cada ano, correspondendo a 15,43 casos a cada 100 mil mulheres, sendo mais incidente nas Regiões Norte, Nordeste e Centro-Oeste, afetando principalmente aquelas de baixo nível socioeconômico e com menor acesso às ações de rastreamento (Lima et al., 2017; INCA, 2019; Davilla et al., 2021).

Dessa forma, a Organização Mundial da Saúde (OMS) buscando prevenir novos caso de câncer cervical, bem como outras patologias oriundas do HPV, passou a recomendar a introdução da vacinação contra o vírus como parte das Políticas Nacionais de Imunização (PNI) (WHO/OMS, 2017; Sintaremi et al., 2020). Além do esquema vacinal, o câncer cervical é passivo de prevenção através do rastreamento regular, relacionado ao teste de Papanicolaou, que tem como finalidade identificar alterações celulares presentes no colo do útero, bem como o HPV que propiciam o risco de desenvolvimento cancerígeno, essa prática tem demostrado uma ótima estratégia para a diminuição dos número de óbitos pela doença, levando em consideração a queda das taxas registradas nos últimos 40 anos referente a morbidade e mortalidade ligadas à neoplasia do colo do útero (Richards et al., 2020).

Ainda que os resultados sejam promissores, há a existência de um número crescente na não-adesão ao exame de citopatológia, reflexo do baixo nível socioeconômico e de escolaridade, ausência de companheiro, temor pelo resultado, constrangimento pela realização do exame, indisponibilidade de horário, dificuldade de acesso à unidade de saúde, hábitos de vida e falta de conhecimento sobre o teste (Lima et al., 2017). A desinformação acerca das causas, sintomas, prevenção e tratamento são consideradas agravantes e fatores que corroboram para a baixa adesão ao rastreamento do câncer de colo uterino, dessa forma, entende-se que há a necessidade de estimular a pactuação de estratégias educacionais que possibilitem a ampliação do conhecimento social acerca da patologia em discussão, visando o grande impacto que essas ações possuem no aumento da busca das mulheres pelo rastreamento da doença (Caster et al., 2017).

Sendo assim, a equipe multiprofissional exerce um papel importante no que tange a propagação de conhecimento sobre a neoplasia do colo do útero, para assim tentar minimizar os fatores e comportamentos de riscos, e estimular a adesão ao esquema vacinal, bem como a prática de rastreamento, acompanhando a disponibilização do exame de acordo com o cenário de cada local (Compaore et al., 2016). Estudo de Wamai et al., (2012), fortalece que o sucesso para a prevenção do câncer de colo do útero é o aumento da disseminação de conhecimento entrelaçado à conscientização pública e a disponibilização de alternativas para o seu tratamento.

Dessa forma, percebe-se a importância de construir conhecimento sobre as boas práticas educacionais na prevenção e no controlo das neoplasias de colo do útero, a fim de fortalecer e servir de subsídio para a elaboração de políticas de saúde voltadas para o eixo temático. Nesse sentido, o presente artigo teve como objetivo descrever a eficácia das ações de educação em saúde no controle e prevenção das neoplasias do colo do útero. 


\section{Metodologia}

O artigo utilizou a metodologia de revisão integrativa da literatura com base qualitativa-descritiva. Para Ercole et al., (2014), a revisão integrativa sistemática é caracterizada como uma forma metodológica que apresenta resultados sintetizados, ordenados e abrangentes de determinado eixo temático, além ao pesquisador a compilação de estudos quase-experimental, e experimental, associando-os com a teorias. Dessa forma, Pereira et al. (2018), descrevem que os estudos qualitativos são de cunho descritivo no qual baseia-se na interpretação do pesquisador a partir de tal fato estudado.

A questão norteadora respondida a partir desta revisão é: "Qual a eficácia das ações de educação em saúde no controle e prevenção das neoplasias do colo do útero?”. Para responder a questão da pesquisa foram utilizados artigos dos seguintes repositórios eletrônicos: National Library of Medicine (PUBMED) e Scientific Eletronic Library Online (SciELO). Para tal, os Descritores em Saúde (DeCS) utilizados foram: Educação em Saúde; Prevenção de Doenças; Controle; Neoplasias do Colo do Útero (versão inglês e português).

A coleta nos repositórios eletrônicos se deram a partir de agosto de 2020 à novembro de 2021, a partir dos achados os artigos foram elegíveis com base na leitura prévia do título e resumo que respondiam a questão da pesquisa, posteriormente, os artigos aprovados na primeira etapa de seleção foram lidos de forma completa, vale ressaltar, que os estudos duplicados foram excluídos. Por fim, foi realizada a extração dos dados relevantes à presente pesquisa.

Para a realização deste artigo foram empregados alguns critérios de elegibilidade, tais como: estudos publicados nos idiomas inglês e português; que compreendesse os últimos cinco anos, ou seja, artigos publicados a partir de 2016; artigos completos e disponibilizados de forma gratuita. De tal forma, foram considerados inelegíveis teses de doutorado e dissertações de mestrado; artigos em outros idiomas que não os aqui já citados e aqueles publicados anteriormente a 2016.

Em um primeiro momento, após o cruzamento dos descritores, foram encontrados 1.390 artigos na plataforma PUBMED e 21 referente à SciELO, totalizando 1.411 artigos. Após a aplicação dos critérios de elegibilidade foram excluídos 1.396 por não atenderam as especificidades empegadas pelo estudo e 01 por duplicação, sendo assim, utilizou-se o total de 14 artigos nesta presente pesquisa (Quadro 1).

Quadro 1. Relação das publicações selecionadas para o estudo.

\begin{tabular}{|c|c|c|c|c|c|c|}
\hline $\mathbf{r}$ & Autores & Título & $\begin{array}{l}\text { Tipo de } \\
\text { Estudo }\end{array}$ & $\begin{array}{l}\text { População do Estudo/ Local } \\
\text { de Estudo }\end{array}$ & Objetivo do Estudo & Principais Resultados \\
\hline A01 & $\begin{array}{l}\text { Kwang, N. B., } \\
\text { Mahayudin, T., } \\
\text { Yien, H. L., } \\
\text { Abdul Karim, A. } \\
\text { K., Teik, C. K., } \\
\text { \& Shan, L. P. } \\
(2016) .\end{array}$ & $\begin{array}{l}\text { Effect of an Educational } \\
\text { Intervention on } \\
\text { Knowledge of Human } \\
\text { Papillomavirus } \\
\text { Vaccination among Pre- } \\
\text { University Students in } \\
\text { Malaysia. }\end{array}$ & $\begin{array}{l}\text { Estudo } \\
\text { experiental }\end{array}$ & pré-universitários; & $\begin{array}{lrrr}\text { Avaliar } & \text { o } & \text { efeito } & \text { da } \\
\text { intervenção educacional no } \\
\text { conhecimento da } & \text { vacinação } \\
\text { contra o } & \text { HPV } & \text { para } & \text { a } \\
\text { prevenção } & \text { do } & \text { câncer } \\
\text { cervical entre } & \text { estudantes } \\
\text { pré-universitários } & \text { na } \\
\text { Malásia. } & \end{array}$ & $\begin{array}{l}\text { O conhecimento sobre a infecção e } \\
\text { vacinação pelo HPV é baixo entre os } \\
\text { pré-universitários. A intervenção } \\
\text { educacional na forma de folhetos } \\
\text { informativos parece ser eficaz na } \\
\text { criação de consciência e na melhoria do } \\
\text { conhecimento. }\end{array}$ \\
\hline A02 & $\begin{array}{l}\text { Musa, } \\
\text { Achenbach, C. J., } \\
\text { O'Dwyer, L. C., } \\
\text { Evans, C. T., } \\
\text { McHugh, M., } \\
\text { Hou, L., Simon, } \\
\text { M. A., Murphy, } \\
\text { R. L., \& Jordan, } \\
\text { N. (2017). }\end{array}$ & $\begin{array}{l}\text { Effect of cervical } \\
\text { cancer education and } \\
\text { provider } \\
\text { recommendation for } \\
\text { screening on screening } \\
\text { rates: A systematic } \\
\text { review and reta- } \\
\text { analysis. }\end{array}$ & $\begin{array}{l}\text { Revisão } \\
\text { sistemática } \\
\text { de cunho } \\
\text { meta-análise }\end{array}$ & $\begin{array}{l}\text { Todas as mulheres } \\
\text { qualificadas para participar de } \\
\text { um programa de rastreamento } \\
\text { do câncer do colo do útero, } \\
\text { incluindo mulheres sem } \\
\text { rastreamento prévio de câncer } \\
\text { do colo do útero e mulheres } \\
\text { com consultas de rastreamento } \\
\text { em dia ou vencidas em vários } \\
\text { locais; Chicago, Estados } \\
\text { Unidos da America. }\end{array}$ & $\begin{array}{l}\text { Compreender a evidência do } \\
\text { efeito da educação sobre o } \\
\text { câncer do colo do útero em } \\
\text { comparação com as } \\
\text { condições de controle nas } \\
\text { taxas de rastreamento do } \\
\text { câncer do colo do útero na } \\
\text { população de mulheres } \\
\text { elegíveis em risco de câncer } \\
\text { do colo do útero. }\end{array}$ & $\begin{array}{l}\text { O uso de intervenções educacionais } \\
\text { baseadas na teoria aumentou } \\
\text { significativamente as taxas de } \\
\text { rastreamento do câncer cervical em } \\
\text { mais do que o dobro. }\end{array}$ \\
\hline
\end{tabular}


Al-Shaikh, G. K., Syed, S. B., \begin{tabular}{l|l} 
Syed, S. B., & education programme: \\
Fayed, A. A., Al- & $\begin{array}{l}\text { Level of knowledge } \\
\text { Shel }\end{array}$
\end{tabular} A., about preventionledge Al-Mussaed, E. $\begin{aligned} & \text { about prevention of } \\ & \text { cervical cancer among }\end{aligned}$ M., Khan, F. H., cervical cancer among \& Elmorshedy, H. N. (2017). education on perception Dim, C., \& screening for cervical intervenção Mulheres em idade Ezeoke, U. (2017).

U. south-east Nigeria: before and after study. Barati, M., Khani Intervention Based on Bashirian S., Theory on Preventing Hazavehei, S. Cervical Cancer among (2018).
Malmir, S. Effect of an Educational

\section{Estudo quase} experimental sexo feminino da bint Abulrahman, em Riade, Arábia Saudita.
Avaliar a eficácia de programa de educação em O programa de educação em saúde foi saúde sobre o conhecimento eficaz na melhoria do nível de do vírus do papiloma conhecimento sobre o papilomavírus humano entre estudantes de humano. medicina.
Avaliar a eficácia da educação de pares em saúde sobre a percepção, a vontade de fazer o rastreamento e a aceitação do rastreamento do câncer do colo do útero por mulheres.

Determinar a eficácia de uma intervenção educacional para prevenir o câncer cervical entre mulheres iranianas marginalizadas com base na Teoria da Motivação de Proteção (PMT) como referencial teórico.

in West Iran.
Estudo quase Mulheres residente (1)

.

.
Y. (2018).
Kim, H. W.,

\section{p}

fem

Mães de adolescentes do sexo feminino; Coreia do Sul.
Após a percepção dos riscos individuais a prática de rastreamento diferença observada estatisticamente significativa.

A prevalência de teste de Papanicolaou regular e encaminhamento para centros de saúde aumentaram significativamente após meses da aplicação da estrategia educativa.

Mostrou um aumento significativo no Examinar os efeitos da conhecimento do teste de educação baseada na Papanicolaou, conhecimento do câncer

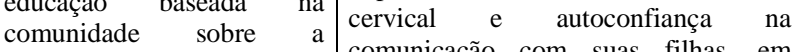
\begin{tabular}{ll|l} 
prevenção do câncer & $\begin{array}{l}\text { comunicação com suas filhas, em } \\
\text { comparação com o grupo de controle. }\end{array}$
\end{tabular} cervical em relação ao No pós-teste, as mães do grupo conhecimento das mães sul- experimental também perceberam o coreanas sobre o teste de exancer cervical como mais ameaçador, Papanicolau (Pap) e o sua filha como mais suscetível ao

\section{|<smiles>C#CC1CCCCC1</smiles>

Mbachu, C., and practice of Estudo de experimental Kermanshah, Irã.

\section{Effect of community-}

Effect of communitybased education to Estudo préKorean mothers in teste-pósrelation to the teste de $\begin{array}{llrl}\text { prevention } & \text { of } & \text { cervical } & \text { grupo } \\ \text { cancer } & \text { in } & \text { their } & \text { controle não }\end{array}$ daughters: A non- randomizado randomized trial.
O estudo teve como sujeito de pesquisa artigos publicados na

Saei Ghare Naz, M., Kariman, N., Ebadi,

A07 Ozgoli, Rashidi Fakari, F. (2018).

\section{Educational} Interventions Cervical \begin{tabular}{r|l} 
for & Revisão \\
Cancer & sistemática \\
ior of & de $\quad$ cunh
\end{tabular}

Screening Behavior of
Women: A Systematic Review. biblioteca Cochrane, Web of Ghasemi, V G.,
Sadoh, A. E., Effect of Peer Okonkwobo, C., Education Nwaneri, D. U., Knowledge of Human Ogboghodo, B. Papilloma Virus and C., Eregiea, C., Cervical Cancer among Oviawe, O., \& Female Adolescent Famuyiwa, O. Students in Benin City, (2018).

Nigeria.

Kocaöz, S., The Effect of Education Özçelik, H., on the Early Diagnosis Talas, MS, of Breast and Cervix A09 Akkaya, F., Cancer on the Women's Özkul, $\quad$ F., $\quad$ Attitudes and Behaviors Kurtuluş, A., \& Regarding Participating Ünlü, F. (2018). $\quad$ in Screening Programs.
Estudo de Adolescentes do sexo intervenção feminino; Benin, Nigéria.

$$
\text { City, }
$$
Science, Science Direct, intervenções educacionais PubMed, Scopus e o no comportamento de mecanismo de busca do rastreamento do câncer Google Scholar sobre câncer cervical (CCS) de mulheres. de colo do útero.

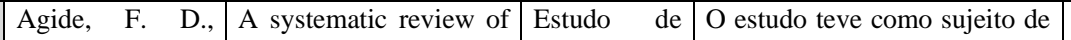
População feminina; Aldeias e Estudo semi- vilas dos distritos Central e experimental Yesilgolcuk do Centro da Província de Niğde. do câncer cervical aumentou $6,8 \%$ e a papilomavírus humano, $\begin{aligned} & \text { sua } \\ & \text { câncer cervical, o exame de }\end{aligned}$ autoconflança na napanicolaou como recomendado para

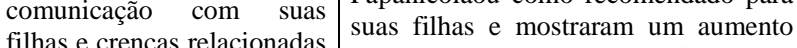
filhas e crenças relacionadas
à saúde sobre suas filhas. $\begin{aligned} & \text { significativo em sua autoeficácia de } \\ & \text { secomendando }\end{aligned}$ recomendando o exame de Papanicolaou para a filha. Diferentes intervenções e estruturas de mudança de comportamento em saúde fornecem uma base eficaz para a prevenção do câncer do colo do útero. Os provedores de saúde podem escolher métodos educacionais com base nas situações particulares do cliente.

O conhecimento sobre o câncer do colo $\begin{array}{lrr}\text { Determinar o efeito da } & \text { do útero, o conhecimento dos fatores } \\ \text { de risco e da causa do câncer do colo }\end{array}$ educação por pares no do útero eram baixos antes do conhecimentor de $\begin{aligned} & \text { do útero eram baixos antes do } \\ & \text { treinamento de pares. Houve melhora }\end{aligned}$ adolescentes do sexo estatisticamente significativa na feminino sobre o HPV, o $\begin{aligned} & \text { estatisticamente significativa } \\ & \text { conscientizaça sobre o câncer do colo }\end{aligned}$ câncer do colo do útero, seu $\begin{aligned} & \text { conscientização } \\ & \text { do útero e nos domínios do }\end{aligned}$

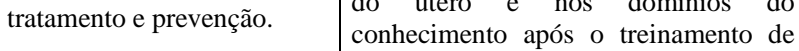

Definir o efeito da educação no diagnóstico precoce do câncer de mama e colo do A educação sobre o diagnóstico útero sobre as atitudes e precoce de câncer de mama e colo do comportamentos das útero teve efeitos positivos sobre os mulheres em relação à comportamentos de saúde das mulheres participação nos programas relacionados ao autoexame das mamas, de rastreamento do Centro mamografia e exames de de Diagnóstico, Triagem e Papanicolaou.

Treinamento do Câncer CEDSTC.

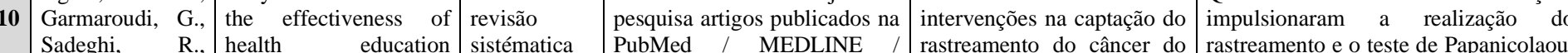
Sadeghi, R., health education sistématica $\quad$ PubMed / MEDLINE / rastreamento do câncer do rastreamento e o teste de Papanicolaou. 


\begin{tabular}{|c|c|c|c|c|c|c|}
\hline & $\begin{array}{l}\text { Shakibazadeh, } \\
\text { E., Yaseri, M., } \\
\text { Koricha, Z. B., \& } \\
\text { Tigabu, B. M. } \\
\text { (2018). }\end{array}$ & $\begin{array}{l}\text { interventions to } \\
\text { increase cervical cancer } \\
\text { screening uptake. }\end{array}$ & & $\begin{array}{l}\text { PubMed Central, EMBASE, } \\
\text { Cochrane Central Register of } \\
\text { Controlled Trials, Web of } \\
\text { Science e Google Scholar } \\
\text { sobre câncer de colo do útero. }\end{array}$ & colo do útero. & $\begin{array}{l}\text { No entanto, as intervenções de } \\
\text { educação em saúde em nível individual } \\
\text { foram priorizadas em muitos dos } \\
\text { estudos. }\end{array}$ \\
\hline A11 & 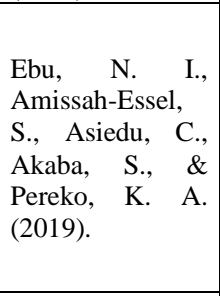 & $\begin{array}{l}\text { Impact of health } \\
\text { education intervention } \\
\text { on knowledge and } \\
\text { perception of cervical } \\
\text { cancer and screening } \\
\text { for women in Ghana. }\end{array}$ & $\begin{array}{l}\text { Estudo de } \\
\text { controle não } \\
\text { equivalente }\end{array}$ & $\begin{array}{l}\text { Mulheres frequentantes de } \\
\text { igrejas ganenses; Região } \\
\text { Central de Gana. }\end{array}$ & $\begin{array}{l}\text { Determinar o efeito da } \\
\text { intervenção de educação em } \\
\text { saúde sobre o câncer } \\
\text { cervical e as percepções do } \\
\text { rastreamento de mulheres } \\
\text { nos distritos de Komenda, } \\
\text { Edina, Eguafo e Abirem } \\
\text { (KEEA) na Região Central } \\
\text { de Gana. }\end{array}$ & $\begin{array}{l}\text { As intervenções de educação em saúde } \\
\text { se mostraram essenciais para melhorar } \\
\text { o conhecimento e as percepções e } \\
\text { aumentar a autoeficácia das mulheres } \\
\text { sobre o câncer cervical e o } \\
\text { rastreamento. }\end{array}$ \\
\hline A12 & \begin{tabular}{lr}
\multicolumn{2}{l}{ Calderón-Mora, } \\
J., Byrd, T. \\
Alomari, \\
Salaiz, & R., \\
Dwivedi, & A., \\
Mallawaarachchi \\
, I., \& Shokar, N. \\
$(2020)$.
\end{tabular} & $\begin{array}{lr}\text { Group } & \text { Versus } \\
\text { Individual Culturally } \\
\text { Tailored and Theory- } \\
\text { Based Education to } \\
\text { Promote } \quad \text { Cervical } \\
\text { Cancer } \quad \text { Screening } \\
\text { Among r the } \\
\text { Underserved Hispanics: } \\
\text { A Cluster Randomized } \\
\text { Trial. }\end{array}$ & $\begin{array}{l}\text { Estudo } \\
\text { controlado } \\
\text { randomizado } \\
\text { por } \\
\text { agrupamento }\end{array}$ & $\begin{array}{l}\text { Mulheres de } 21 \text { à } 65 \text { anos; } \\
\text { Condados de El Paso e } \\
\text { Hudspeth, Texas. }\end{array}$ & $\begin{array}{l}\text { Determinar se a educação } \\
\text { em grupo é tão eficaz } \\
\text { quanto a educação } \\
\text { individual para melhorar o } \\
\text { rastreamento do câncer do } \\
\text { colo do útero ao longo da } \\
\text { fronteira EUA-México. }\end{array}$ & $\begin{array}{l}\text { Aumentos significativos entre a } \\
\text { educação do grupo no } \\
\text { acompanhamento para conhecimento, } \\
\text { suscetibilidade percebida, seriedade } \\
\text { percebida e normas subjetivas e } \\
\text { diminuição significativa para } \\
\text { benefícios percebidos. }\end{array}$ \\
\hline A13 & $\begin{array}{lr}\text { Romli, } & \text { R., } \\
\text { Shahabudin, } & \text { S., } \\
\text { Saddki, N., } & \& \\
\text { Mokhtar, } & \text { N. } \\
(2020) . & \end{array}$ & $\begin{array}{lr}\text { Effectiveness } & \text { of a } \\
\text { Health } & \text { Education } \\
\text { Program to Improve } \\
\text { Knowledge } & \text { and } \\
\text { Attitude } & \text { Towards } \\
\text { Cervical Cancer and } \\
\text { Pap Smear: } \quad \text { A } \\
\text { Controlled Community } \\
\text { Trial in Malaysia. }\end{array}$ & $\begin{array}{l}\text { Ensaio } \\
\text { clínico } \\
\text { controlado }\end{array}$ & $\begin{array}{l}\text { Mulheres residentes nos } \\
\text { distritos de Alor Setar e } \\
\text { Sungai Petani; Malásia. }\end{array}$ & $\begin{array}{l}\text { Examinar a eficácia de um } \\
\text { programa de educação em } \\
\text { saúde para melhorar } \\
\text { o conhecimento e atitude em } \\
\text { relação ao câncer cervical e } \\
\text { teste de Papanicolaou em } \\
\text { Kedah, um estado do norte } \\
\text { da Malásia. }\end{array}$ & $\begin{array}{l}\text { O conhecimento sobre o câncer } \\
\text { cervical e o esfregaço de Papanicolaou } \\
\text { e a atitude em relação ao exame de } \\
\text { Papanicolaou entre as mulheres nos } \\
\text { grupos de intervenção e controle } \\
\text { melhoraram significativamente. A } \\
\text { realização do Papanicolaou esfregaço } \\
\text { no grupo de intervenção aumentou } \\
\text { significativamente de } 48,0 \% \text { na linha } \\
\text { de base para } 68,0 \% \text {. }\end{array}$ \\
\hline A14 & $\begin{array}{lr}\text { Eghbal, S. } & \text { B., } \\
\text { Karimy, } & \text { M., } \\
\text { Kasmaei, } & \text { P., } \\
\text { Roshan, Z. } & \text { A., } \\
\text { Valipour, R., \& } \\
\text { Attari, S. } & \text { M. } \\
(2020) . & \end{array}$ & $\begin{array}{l}\text { Evaluating the effect of } \\
\text { an educational program } \\
\text { on increasing cervical } \\
\text { cancer screening } \\
\text { behavior among rural } \\
\text { women in Guilan, Iran. }\end{array}$ & $\begin{array}{l}\text { Estudo quase } \\
\text { experimental }\end{array}$ & $\begin{array}{l}\text { Mulheres rurais do norte do } \\
\text { Irã; Guilan, Irã. }\end{array}$ & $\begin{array}{l}\text { Investigar os efeitos da } \\
\text { intervenção educativa } \\
\text { baseada no Modelo de } \\
\text { Crenças em Saúde (HBM) } \\
\text { na realização do exame de } \\
\text { Papanicolaou em mulheres } \\
\text { rurais do norte do Irã. }\end{array}$ & $\begin{array}{l}\text { Houve diferença significativa nos } \\
\text { escores médios de desempenho do } \\
\text { conhecimento e todos os construtos do } \\
\text { HBM nos dois grupos }(p<0,001) \text {. A } \\
\text { taxa de realização do teste de } \\
\text { Papanicolaou no grupo experimental } \\
\text { aumentou de } 18,7 \text { para } 78,7 \% \text { no grupo } \\
\text { de intervenção. }\end{array}$ \\
\hline
\end{tabular}

Fonte: Tiago et al. (2021).

Nesse sentido, ressalta-se que o presente artigo atende todas as normas propostas pelo Conselho Nacional de Saúde através da portaria 466/2012.

\section{Resultados e Discussão}

Diferentes estudos empregam meios de comunicação heterogênicos para fomentar o conhecimento sobre o câncer cervical, bem como seus riscos, manifestações clínicas, tratamento e prevenção. A partir do levantamento realizado por Saei Ghare Naz et al., (2018), grande parte dos estudos analisados apresentaram ligações telefônicas, apresentação em slides, discussões grupais, palestras informativas, aconselhamentos, entre outros métodos, como eficientes na sensibilização da importância do rastreamento do câncer de colo do útero e da vacinação contra o HPV.

O estudo de Romli et al., (2020) utilizou distribuição de panfletos, envio de SMS e palestras informativas como métodos educacionais, evidencia que a forma mais eficiente após análise comparativa das metodologias aqui já citadas foi a palestra educacional, apresentando melhores resultados. Os autores ainda descrevem uma crescente no que refere ao nível de conhecimentos e na mudança de atitudes nas mulheres participantes após a implantação das palestras.

Por outro lado, Musa et al., (2017), descrevem que a utilização da combinação entre telefonemas, carta-convite e carta de nomeação corroboram para o aumento da procura das mulheres pelo rastreamento do câncer de colo do útero, no entanto, apesar de demonstrarem bons resultados, a junção desses métodos apresenta fragilidade levando em consideração as baixas 
taxas de alfabetização. Por tanto, ressalta-se a importância da realização de um estudo de campo analisando as fragilidades e vulnerabilidade da população local.

Nesse sentido, é possível observar uma disparidade no acesso ao rastreamento do câncer de colo do útero relacionado ao perfil sociodemográficos, tendo em vista que mulheres empregadas, casadas e com ensino superior são mais propensas a realização do exame citopatológico, isso se deve, ao acesso facilitado dessas mulheres a informações, assim como ao serviço de saúde preventivo, além de contarem com o apoio financeiro e emocional de seus parceiros (Mbachu, et al., 2017).

Estudo de Eghbal et al., (2020), realizado em uma Zona Rural de Guilan no Irã que contou com 160 mulheres, sendo 90\% das participantes predominantemente analfabetas, utilizou como recurso educacional palestras com suporte de slides, panfletos, pôster, filmes e quiz, divididos em três sessões, demonstrou um aumento registrado em mais de $80 \%$ na procura pelas participantes ao método de rastreamento do câncer de colo do útero, além desses efeitos, foi possível observar que grande parte das mulheres tiverem um crescente desenvolvimento da consciência e do desempenho. Há estudos que relacionam o desenvolvimento de consciência com a maior adesão ao exame de Papanicolaou, bem como favorece a mudança positiva de crenças, atitudes e comportamentos (Kocaöz et al., 2018; Eghbal et al., 2020).

Essa afirmação pode ser evidenciada pelo estudo de Ebu et al., (2019), que demonstrou a partir da inserção da intervenção educacional, que as participantes desenvolveram uma consciência mais crítica, corroborando para a melhora no que tange a percepção da susceptibilidade a patologia em discussão, sendo esse achado, reflexo da autoavaliação de risco ao câncer do colo do útero. Além disso, os autores relatam um aumento significativo na percepção das mulheres quanto aos benefícios do rastreamento do câncer cervical.

Em um estudo que buscou avaliar a eficácia de um programa de educação em saúde com suporte de vídeos sobre o HPV, evidenciou que após a implementação das ações educativas houve um aumento de conhecimento expressivo sobre medidas preventivas, fatores de risco, manifestações clinicas e detecção da doença, além de que, o estudo descreve que a utilização de recursos audiovisual, tais como: filmes e vídeos, são estratégias promissoras, haja visto o despertar da atenção e do interesse do público presente (Al-Shaikh et al., 2017).

Outro estudo buscou avaliar a eficácia das ações de educação em saúde grupais e individuais, demonstrou que as educações em saúde realizadas em grupos são mais eficientes para a conscientização sobre o rastreamento do câncer cervical se comparada as ações individuais (Calderón-Mora et al., 2020). Nessa mesma perspectiva, Kwang et al., (2016), descrevem em seu estudo que as ações educativas em grupos associadas a entrega de folhetos informativos pode ser uma estratégia benéfica principalmente na população mais jovem, levando em consideração o baixo conhecimento desse grupo sobre infecção pelo HPV e neoplasia do colo do útero.

Frente ao exposto, atividades educacionais de sensibilização acerca de aspectos gerais que tratem sobre o câncer cervical e a infecção pelo HPV, também são efetivas para mães de adolescentes, tendo em vista que a partir da aquisição de conhecimento por elas pode melhorar a comunicação e relação mãe-filha, além de servir de subsidio para prevenir o desenvolvimento de tal patologia por meio do rastreamento precoce da neoplasia de colo do útero, assim como para a adesão a vacinação contra o HPV (Kim et al., 2018).

Levantar a pauta sobre o câncer de colo cervical associado ao HPV para a população adolescente, torna-se importante em decorrência da vulnerabilidade deste grupo, relacionado a falta de conhecimento por eles refletido na taxa de $90 \%$ das entrevistadas de uma pesquisa realizada Nigéria que apresentaram não saber que o HPV é uma das causas para o desenvolvimento do câncer do colo do útero, assim como demonstraram baixo conhecimento sobre os fatores de risco para a infecção pelo vírus e outras Infecções Sexualmente Transmissíveis (IST) (Sadoh et al., 2018).

Para Malmir et al., (2018), a Teoria da Motivação de Proteção pode ser uma estratégia benéfica para as ações de educação em saúde, pois a partir dela pode-se avaliar o comportamento de proteção e os fatores que afetam os motivos. Os 
autores descrevem que a teoria associada as intervenções educativas apresentam eficientes resultados na prevenção do câncer de colo do útero e na adesão ao exame de Papanicolaou principalmente em mulheres habitantes de regiões marginalizadas. Dessa forma, Agide et al., (2018), descrevem que quase todas as intervenções de educação em saúde aplicadas a sensibilização do câncer do colo do útero são iniciativas eficazes para aumentar a aceitação absoluta do rastreamento do câncer cervical e a intenção de fazer o rastreamento, além disso, os autores relatam que essas ações ajudam na prevenção de doenças e na mudança de comportamento.

\section{Considerações Finais}

Assim, as ações de educação em saúde tornam-se uma estratégia muito promissora, haja visto, que é uma atividade de baixo custo que permite a difusão de informações e conhecimento para a população em amplo aspecto, corroborando para o aumento da procura daquelas que recebem as ações de educação pela vacinação contra o HPV e a realização do exame de Papanicolaou.

Na confecção deste presente artigo, foi possível perceber a baixa existência de produções que trabalhem essa temática no Brasil, o que nos motiva a poder servir de base para que haja a elaboração de novos estudos que busquem implementar novas formas metodológicas para sensibilização do tema, ou até avaliar a eficácia dos métodos já existentes. Além disso, espera-se o fortalecimento do Sistema Único de Saúde e servir de embasamento para novas políticas públicas de saúde a partir da publicação desta pesquisa.

\section{Referências}

Agide, F. D., Garmaroudi, G., Sadeghi, R., Shakibazadeh, E., Yaseri, M., Koricha, Z. B., \& Tigabu, B. M. (2018). A systematic review of the effectiveness of health education interventions to increase cervical cancer screening uptake. European journal of public health, 28(6), 1156-1162. https://doi.org/10.1093/eurpub/cky197.

Al-Shaikh, G. K., Syed, S. B., Fayed, A. A., Al-Shaikh, R. A., Al-Mussaed, E. M., Khan, F. H., \& Elmorshedy, H. N. (2017). Effectiveness of health education programme: Level of knowledge about prevention of cervical cancer among Saudi female healthcare students. JPMA. The Journal of the Pakistan Medical Association, 67(4), 513-520.

Calderón-Mora, J., Byrd, T. L., Alomari, A., Salaiz, R., Dwivedi, A., Mallawaarachchi, I., \& Shokar, N. (2020). Group Versus Individual Culturally Tailored and Theory-Based Education to Promote Cervical Cancer Screening Among the Underserved Hispanics: A Cluster Randomized Trial. American journal of health promotion : AJHP, 34(1), 15-24. https://doi.org/10.1177/0890117119871004.

Caster, M. M., Norris, A. H., Butao, C. et al. (2017). Assessing the Acceptability, Feasibility, and Effectiveness of a Tablet-Based Cervical Cancer Educational Intervention. J Canc Educ 32, 35-42. https://doi.org/10.1007/s13187-015-0953-6.

Compaore, S., Ouedraogo, C., Koanda, S., Haynatzki, G., Chamberlain, R. M., \& Soliman, A. S. (2016). Barriers to Cervical Cancer Screening in Burkina Faso: Needs for Patient and Professional Education. Journal of cancer education: the official journal of the American Association for Cancer Education, 31(4), 760-766. https://doi.org/10.1007/s13187-015-0898-9.

Davilla, M. de S. D. et al. (2021). Objeto virtual de aprendizagem sobre rastreamento do câncer do colo do útero. Acta Paulista de Enfermagem 34, https://doi.org/10.37689/acta-ape/2021AO00063. https://doi.org/10.37689/acta-ape/2021AO00063.

Ebu, N. I., Amissah-Essel, S., Asiedu, C., Akaba, S., \& Pereko, K. A. (2019). Impact of health education intervention on knowledge and perception of cervical cancer and screening for women in Ghana. BMC public health, 19(1), 1505. https://doi.org/10.1186/s12889-019-7867-x.

Eghbal, S. B., Karimy, M., Kasmaei, P., Roshan, Z. A., Valipour, R., \& Attari, S. M. (2020). Evaluating the effect of an educational program on increasing cervical cancer screening behavior among rural women in Guilan, Iran. BMC women's health, 20(1), 149. https://doi.org/10.1186/s12905-020-01020-7.

Ercole, F. F., Melo, L. S., \& Alcoforado, C. L. G. C. (2014). Revisão integrativa versus revisão sistemática. Rev Min Enferm., 18(1): 1-260. http://www.dx.doi.org/10.5935/1415-2762.20140001.

INCA. Instituto Nacional de Câncer José Alencar Gomes da Silva. (2018). ABC do câncer: abordagens básicas para o controle do câncer. 4. ed. rev. atual. INCA. Instituto Nacional de Câncer José Alencar Gomes da Silva. (2019). Estimativa 2020: incidência de câncer no Brasil.

Kim, H. W., Park, S., \& Kim, Y. (2018). Effect of community-based education to Korean mothers in relation to the prevention of cervical cancer in their daughters: A non-randomized trial. Japan journal of nursing science: JJNS, 15(2), 146-155. https://doi.org/10.1111/jjns.12179.

Kocaöz, S., Özçelik, H., Talas, M. S., Akkaya, F., Özkul, F., Kurtuluş, A., \& Ünlü, F. (2018). The Effect of Education on the Early Diagnosis of Breast and Cervix Cancer on the Women's Attitudes and Behaviors Regarding Participating in Screening Programs. Journal of cancer education: the official journal of the American Association for Cancer Education, 33(4), 821-832. https://doi.org/10.1007/s13187-017-1193-8. 
Kwang, N. B., Mahayudin, T., Yien, H. L., Abdul Karim, A. K., Teik, C. K., \& Shan, L. P. (2016). Effect of an Educational Intervention on Knowledge of Human Papillomavirus Vaccination among Pre-University Students in Malaysia. Asian Pacific journal of cancer prevention: APJCP, 17(1), 267-274. https://doi.org/10.7314/apjcp.2016.17.1.267.

Lima, T. M. et al. (2017). Telephone interventions for adherence to colpocytological examination1 1 Paper extracted from Doctoral Dissertation "Intervenções por telefone para adesão ao exame colpocitológico", presented to Universidade Federal do Ceará, Fortaleza, CE, Brazil. Revista Latino-Americana de Enfermagem [online].

Malmir, S., Barati, M., Khani Jeihooni, A., Bashirian, S., \& Hazavehei, S. (2018). Effect of an Educational Intervention Based on Protection Motivation Theory on Preventing Cervical Cancer among Marginalized Women in West Iran. Asian Pacific journal of cancer prevention: APJCP, 19(3), 755-761. https://doi.org/10.22034/APJCP.2018.19.3.755.

Mbachu, C., Dim, C., \& Ezeoke, U. (2017). Effects of peer health education on perception and practice of screening for cervical cancer among urban residential women in south-east Nigeria: a before and after study. BMC women's health, 17(1), 41. https://doi.org/10.1186/s12905-017-0399-6.

Musa, J., Achenbach, C. J., O'Dwyer, L. C., Evans, C. T., McHugh, M., Hou, L., Simon, M. A., Murphy, R. L., \& Jordan, N. (2017). Effect of cervical cancer education and provider recommendation for screening on screening rates: A systematic review and meta-analysis. PloS one, 12(9), e0183924. https://doi.org/10.1371/journal.pone.0183924.

Pereira A. S. et al. (2018). Metodologia da pesquisa científica. UFSM. https://repositorio.ufsm.br/bitstream/handle/1/15824/Lic_Computacao_MetodologiaPesquisa-Cientifica.pdf?sequence $=1$.

Richards, C., Thomas-Purcell, K. B., Vemulapalli, K. C. et al. (2020). Woman to Woman: Implementation of a Cervical Cancer Education Training Program for Grenadian Lay Health Advisors. J Canc Educ 35, 557-562. https://doi.org/10.1007/s13187-019-01495-5.

Romli, R., Shahabudin, S., Saddki, N., \& Mokhtar, N. (2020). Effectiveness of a Health Education Program to Improve Knowledge and Attitude Towards Cervical Cancer and Pap Smear: A Controlled Community Trial in Malaysia. Asian Pacific journal of cancer prevention: APJCP, 21(3), 853-859. https://doi.org/10.31557/APJCP.2020.21.3.853.

Sadoh, A. E., Okonkwobo, C., Nwaneri, D. U., Ogboghodo, B. C., Eregiea, C., Oviawe, O., \& Famuyiwa, O. (2018). Effect of Peer Education on Knowledge of Human Papilloma Virus and Cervical Cancer among Female Adolescent Students in Benin City, Nigeria. Annals of global health, 84(1), 121-128. https://doi.org/10.29024/aogh.24.

Saei Ghare Naz, M., Kariman, N., Ebadi, A., Ozgoli, G., Ghasemi, V., \& Rashidi Fakari, F. (2018). Educational Interventions for Cervical Cancer Screening Behavior of Women: A Systematic Review. Asian Pacific journal of cancer prevention: APJCP, 19(4), 875-884. https://doi.org/10.22034/APJCP.2018.19.4.875.

Sitaresmi, M. N., Rozanti, N. M., Simangunsong, L. B. et al. (2020). Improvement of Parent's awareness, knowledge, perception, and acceptability of human papillomavirus vaccination after a structured-educational intervention. BMC Public Health 20, https://doi.org/10.1186/s12889-020-09962-1.

Wamai, R. G., Ayissi, C. A., Oduwo, G. O., Perlman, S., Welty, E., Manga, S., \& Ogembo, J. G. (2012). Assessing the effectiveness of a community-based sensitization strategy in creating awareness about HPV, cervical cancer and HPV vaccine among parents in North West Cameroon. Journal of community health, 37(5), 917-926. https://doi.org/10.1007/s10900-012-9540-5.

WHO/OMS. World Health Organization. (2017). Human papillomavirus vaccines: WHO position paper, may 2017-recommendations. Vaccine. 35:5753-5. https://doi.org/10.1016/j.vaccine.2017.05.069. 